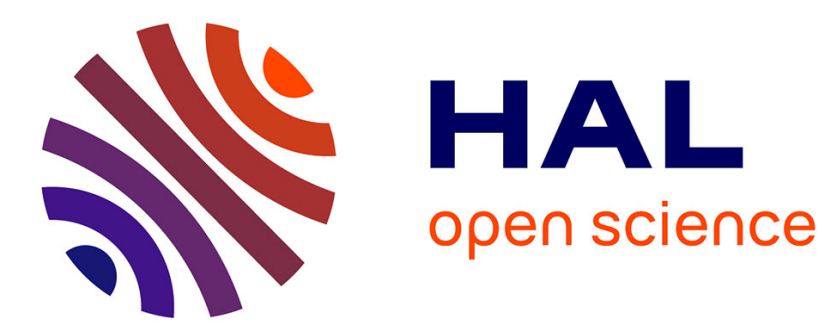

\title{
L'expérience comme promesse et comme détresse chez Karl Barth
}

\author{
Anthony Feneuil
}

\section{To cite this version:}

Anthony Feneuil. L'expérience comme promesse et comme détresse chez Karl Barth. Transversalités, 2019, nº149 (2), pp.87-102. 10.3917/trans.149.0085 . hal-02110679

\section{HAL Id: hal-02110679 \\ https://hal.univ-lorraine.fr/hal-02110679}

Submitted on 10 May 2019

HAL is a multi-disciplinary open access archive for the deposit and dissemination of scientific research documents, whether they are published or not. The documents may come from teaching and research institutions in France or abroad, or from public or private research centers.
L'archive ouverte pluridisciplinaire HAL, est destinée au dépôt et à la diffusion de documents scientifiques de niveau recherche, publiés ou non, émanant des établissements d'enseignement et de recherche français ou étrangers, des laboratoires publics ou privés. 


\section{L'EXPÉRIENCE COMME PROMESSE ET COMME DÉTRESSE CHEZ KARL BARTH \\ Anthony Feneuil}

Institut Catholique de Paris | «Transversalités »

2019/2 nº 149 | pages 85 à 102

ISSN 1286-9449

Article disponible en ligne à l'adresse :

https://www.cairn.info/revue-transversalites-2019-2-page-85.htm

Distribution électronique Cairn.info pour Institut Catholique de Paris.

(C) Institut Catholique de Paris. Tous droits réservés pour tous pays.

La reproduction ou représentation de cet article, notamment par photocopie, n'est autorisée que dans les limites des conditions générales d'utilisation du site ou, le cas échéant, des conditions générales de la licence souscrite par votre établissement. Toute autre reproduction ou représentation, en tout ou partie, sous quelque forme et de quelque manière que ce soit, est interdite sauf accord préalable et écrit de l'éditeur, en dehors des cas prévus par la législation en vigueur en France. Il est précisé que son stockage dans une base de données est également interdit. 


\section{CHRONIQUE}

Chaire de Philosophie et de Théologie Dominique Dubarle

2017-2018

Première partie 


\title{
L'EXPÉRIENCE COMME PROMESSE ET COMME DÉTRESSE CHEZ KARL BARTH ${ }^{1}$
}

\author{
Anthony FENEUIL \\ Université de Lorraine \\ Écritures - EA 3943
}

\section{Un mot qui promet beaucoup}

L'expérience, écrit Hermann Cohen, est un mot « qui promet beaucoup [Vielversprechende] ». On devrait ajouter, empruntant à Barth une expression qu'il affectionne: cette promesse est aussi sa détresse ${ }^{2}$. Elle est si grande, en effet, qu'elle ne peut être tenue. C'est sans doute à cause d'elle que, comme le remarque Cohen, le mot « expérience » est resté un slogan ${ }^{3}$

1. Les articles des Professeurs Anthony Feneuil et Philippe Nouzille ici publiés sont issus des interventions qu'ils ont effectuées les jeudis 14 décembre 2017 et 18 janvier 2018 dans le cadre de la Chaire de Philosophie et de Théologie Dominique Dubarle. Cette Chaire de l'Institut Catholique de Paris porte sur les fondements philosophiques du discours théologique et, de manière plus générale, sur les relations entre philosophie et théologie. Fondée en 2011, cette Chaire - dont le premier titulaire a été le Professeur Jean-Luc Marion de l'Académie française (2011-2016) - est aujourd'hui confiée au Professeur Vincent Holzer. Des contributions des Professeurs David Sendrez et Vincent Holzer lors de la même année académique feront l'objet d'une publication prochaine dans la revue Transversalités.

2. L'association Verheissung/Bedrängnis apparaît plusieurs fois dans les conférences des années 1920 réunies dans le recueil de Karl BARTH, Das Wort Gottes und die Theologie, Munich, Chr. Kaiser Verlag, 1925; Parole de Dieu et parole humaine, trad. Pierre Maury et Auguste Lavanchy, Paris, «Je sers »; Genève, Labor et Fides, 1933. Une fois n'est pas coutume, la traduction française du couple est plus heureuse encore que la version originale.

3. Hermann CoHen, Kants Theorie der Erfahrung, Hildesheim, Olms, 1987, p. 84-85; La théorie kantienne de l'expérience, trad. Éric Dufour et Julien Servois, Paris, Cerf, 2001, p. 106-107. 
parmi les philosophes modernes, revendiqué par tous mais rarement défini précisément, sous peine de dissiper les espoirs qu'il suscitait. Que prometelle donc, l'expérience, de si exorbitant qu'elle ne puisse le donner? Un donné, justement, c'est-à-dire la garantie pour la pensée de ne pas rester seule avec elle-même. Suivant une étymologie bien connue, l'expérience promet une traversée vers le monde, ou une percée du monde en nous, grâce à laquelle nos théories et nos concepts pourraient n'être pas seulement des fantasmes mais des connaissances. Et c'est au nom de cette promesse qu'elle se substitue à la raison, autre slogan d'après Cohen, quand l'exercice métaphysique purement rationnel devient suspect.

À une telle promesse, la théologie n'est pas restée insensible. Comme la philosophie, et peut-être plus encore, elle a adopté le slogan de l'expérience. N'était-elle pas spécialement sujette au risque de construire des théories déconnectées de tout objet réel? Face aux critiques positivistes ou kantiennes qui mettaient en cause la possibilité d'accéder à Dieu sur une base strictement rationnelle, il était certainement inévitable d'en appeler à l'expérience. Dès la fin du XVIII ${ }^{\mathrm{e}}$ siècle et en particulier dans la nébuleuse que l'on appelle le protestantisme libéral, se sont développées des théologies explicitement appliquées à se fonder empiriquement. Un mouvement similaire s'est produit dans la théologie catholique, notamment lors de la crise moderniste, qui d'ailleurs éclate à l'occasion d'une réponse catholique à l'un des principaux représentants du protestantisme libéral de l'époque, Adolf von Harnack ${ }^{4}$. Or si cette réponse et ses suites furent l'objet des attaques magistérielles, c'est justement parce que du point de vue romain, très bien exprimé dans l'encyclique Pascendi en 1907, elles faisaient la part trop belle à l'expérience. Car si la vérité de la doctrine est fondée sur l'expérience, penset-on à Rome, n'est-ce pas toute doctrine qui pourrait être vraie, dès lors qu'elle exprimerait une expérience ${ }^{5}$ ? Il serait pourtant réducteur de limiter l'importance de la notion d'expérience à ces théologies inscrites dans le

4. Le livre d'Alfred LoIsY, L'Évangile et l'Église (1902, dans Alfred Loisy, présenté par Gérard Mordillat et Jérôme Prieur, Paris, Noesis, 2001), se présente en effet comme une réponse aux conférences de Harnack réunies sous le titre: Das Wesen des Christentums, Leipzig, J. C. Hinrichs, 1901 (L'essence du christianisme, trad. Jean-Marc Tétaz, Genève, Labor et Fides, 2015).

5. Voir l'encyclique de Pie X, Pascendi Dominici Gregis: « la doctrine de l'expérience, jointe à l'autre du symbolisme, consacre comme vraie toute religion, sans en excepter la religion païenne. Est-ce qu'on ne rencontre pas dans toutes les religions, des expériences de ce genre? Beaucoup le disent. Or, de quel droit les modernistes dénieraient-ils la vérité aux expériences religieuses qui se font, par exemple, dans la religion mahométane? » 
sillage du protestantisme libéral. Karl Barth lui-même, que l'on présente non sans raison comme un adversaire de ces théologies de l'expérience, non seulement ne renonce pas à l'usage de la notion, mais ne renonce même pas à en faire la clef de la connaissance théologique. Au contraire, il affirme à plusieurs reprises ${ }^{6}$ et au besoin en se référant directement à Schleiermacher qu'une connaissance qui ne serait pas en même temps une expérience, c'est-à-dire qui n'engagerait pas existentiellement le sujet qui la formule, ne pourrait prétendre être véritablement théologique. C'est le sens, souvent mal interprété, de l'ajout de l'adjectif « ecclésiale [kirchliche]» au titre de son grand-œuvre, la Dogmatique ecclésiale. S'il ne peut y avoir de dogmatique qu'ecclésiale, c'est d'abord parce que le critère de la vérité théologique ne peut être indépendant de sa mise à l'épreuve dans la communauté de celles et ceux à qui elle s'adresse. Communauté non seulement humaine, mais Église dans la mesure où elle doit répondre à et d'un envoi de Dieu - la dogmatique ayant pour charge de corriger les discours tenus dans l'Église sur cet envoi. Ainsi chez Barth lui-même, l'expérience tient la plus haute place: elle est en dernier ressort ce qui garantit que nos concepts dogmatiques ne soient pas seulement les nôtres, mais qu'ils soient mis au service de Dieu à travers ce qu'il demande à l'Église. Car il n'y a pas pour lui de connaissance théologique indépendamment de la manière dont cette connaissance engage concrètement nos vies relativement à Dieu, c'est-à-dire dans l'Église.

Voilà donc la promesse de l'expérience: rien de moins que la vérité, l'établissement d'un lien entre la pensée et le réel, que seul un donné peut assurer quand l'exercice de la raison ne semble plus suffire. Mais cette promesse, disais-je, est aussi sa détresse. Elle rend certainement la notion d'expérience inévitable, mais elle la rend aussi encombrante, tout spécialement dans cette tradition kantienne où de plus en plus la confiance dans la

6. Et d'abord lorsqu'il définit dans sa Dogmatique la connaissance de la Parole de Dieu comme une expérience de cette parole. Voir Karl BARTH, Die Kirchliche Dogmatik, Zürich, TVZ, 1980, p. 206; Dogmatique, trad. Fernand Ryser et Jacques de Sénarclens, Genève, Labor et Fides, 1953-1969 [désormais KD], I/1* (l'astérisque renvoie au tome de l'édition en français), § 6, p. 192. Il s'agit d'une des thèses de Barth qui reste constante durant son parcours, depuis L'Épitre aux Romains de 1922 jusqu'aux derniers textes.

7. Karl BARTH, « Nachwort », dans Friedrich D. E. SCHLEIERMACHER, SchleiermacherAuswahl, éd. par Heinz Bolli, Munich, Siebenstern-Taschenbuch-Verlag, 1968; « Postface sur Friedrich D. E. Schleiermacher » (1968), trad. Lore Jeanneret dans La théologie protestante au dix-neuvième siècle, Genève, Labor et Fides, 1969, p. 452. Schleiermacher est souvent considéré comme le fondateur du protestantisme libéral. 
possibilité d'un véritable donné est contestée. L'embarras est encore visible à l'autre bout du siècle, chez Jean-Luc Marion, où il est spécialement mis en scène. À propos du mode d'apparition de ce qu'il appelle le « phénomène saturé ", et dont on peut sans doute dire qu'il s'agit là du donné par excellence, Jean-Luc Marion ne parle pas d'expérience mais de contreexpérience $^{8}$. Derrière le jeu de mots, apparaît une double impossibilité: celle d'abandonner entièrement la notion d'expérience (une contre-expérience, dans le langage courant, c'est évidemment une expérience), tout autant que celle de l'assumer pleinement (le " contre » de la contre-expérience ne désigne pas une expérience qui viendrait en contredire une autre, mais l'opposition à l'expérience en tant que telle). Comme si la réalisation de la promesse - intenable, mais comment y renoncer? - de l'expérience ne pouvait se réaliser que paradoxalement dans l'abandon de l'expérience.

L'intérêt de la pensée de Barth sur ce point est d'avoir affronté directement cette dualité dans le concept d'expérience, et d'avoir non pas cherché à l'atténuer ou à la dépasser, mais au contraire à l'amplifier au maximum pour en faire le cœur de l'expérience par excellence qu'est pour lui la foi. C'est en quoi sa pensée est doublement utile: philosophiquement, parce qu'elle pousse à ses extrémités un problème qui affleure dans la plupart des pensées postkantiennes, et théologiquement, parce que cette exacerbation du problème philosophique de l'expérience lui permet de proposer une pensée originale et provocatrice de l'expérience de la foi.

\section{La radicalisation barthienne de la critique du donné}

Pour comprendre la manière dont il le fait, il faut revenir sur cette détresse qui caractérise la notion d'expérience, c'est-à-dire sur les raisons qui la rendent incapable de tenir ses promesses. D'où vient-elle? Répétonsle: ce que promet l'expérience, c'est un donné. Or la difficulté est évidemment de savoir où exactement situer le lieu de la donation, celui où le réel serait vraiment rencontré et où nos théories pourraient s'y heurter pour être départagées. Cela n'a bien sûr rien d'évident, au point que sans doute une grande partie des débats philosophiques du $\mathrm{XX}^{\mathrm{e}}$ siècle, tant dans la tradition analytique que continentale, pourraient être lus à l'aune de cette question : qu'est-ce qui, dans l'expérience, n'est pas construit mais donné?

8. Jean-Luc MARION, Étant donné, Paris, PUF, 1997, p. 300. 
Évidemment, poser cette question n'implique pas d'y répondre de la manière la plus radicale, en niant purement et simplement la possibilité d'identifier quoi que ce soit dans l'expérience qui ne soit pas construit, même si l'on se situe dans une tradition kantienne, et d'autant moins que l'on est plus rigoureusement kantien. Chez Kant, en effet, il y a bien du donné9 ${ }^{9}$ Certes, l'expérience est structurée formellement, c'est-à-dire construite par les catégories a priori de notre entendement et les formes de l'intuition sensible, mais la matière de l'intuition est bien donnée.

Bruce McCormack, l'un des plus importants commentateurs récents de Karl Barth, a d'ailleurs proposé d'utiliser cette opposition kantienne entre un donné de l'intuition et des formes par lesquelles on la structurerait, pour comprendre l'entreprise de Barth ${ }^{10}$. On aurait affaire à un « réalisme critique » c'est-à-dire à une théologie fondée sur un véritable donné (réalisme), l'autocommunication de Dieu, sa Parole, mais qui serait par principe, en tant que matière, irréductible aux formes conceptuelles que nous utilisons pour le comprendre, et qu'il nous faudrait donc sans cesse réviser (critique). Cette lecture de la conception barthienne de l'expérience est tout à fait éclairante, à condition de ne pas y voir la solution du problème, mais uniquement son énoncé. Car si la théologie doit se fonder sur un donné qu'elle met en forme, celui de la révélation de Dieu, B. McCormack reconnaît en même temps qu'on ne saurait, selon Barth, énoncer les critères permettant d'identifier l'expérience de ce donné ${ }^{11}$. Par conséquent - et B. McCormack le reconnaît volontiers sa notion de réalisme critique n'est pas un modèle épistémologique complet, mais ne tient que sur la reconnaissance du miracle d'après lequel il y a un donné. En d'autres termes, s'il s'agit de kantisme, c'est un kantisme bien particulier, puisque c'est seulement par l'action de Dieu, par miracle ${ }^{12}$, qu'il y a une matière de l'intuition. D'où le fait que le « réalisme critique » est plutôt l'énoncé du problème de l'expérience chez Barth que sa solution: il faut que la connaissance de Dieu soit fondée sur l'expérience d'un donné qu'aucune expérience ne peut donner puisqu'il s'agit d'un miracle. Il reste donc à savoir ce que veut dire ce miracle et en quoi il n'est pas une expérience - ce qui implique de réfléchir à la notion d'expérience elle-même.

9. H. Cohen en est parfaitement conscient et c'est pourquoi il déclare que Kant lui-même ne s'est pas tout à fait libéré de l'expérience comme slogan.

10. Bruce McCORMACK, Karl Barth's Critically Realistic Dialectical Theology, Oxford, Oxford University Press, 1997, p. 129.

11. Ibid., p. 249.

12. Ibid., p. 465. 
Mais d'où vient, d'abord, cette impossibilité pour la révélation de se donner dans une expérience? Il faut revenir sur le cadre épistémologique dans lequel se situe Barth, qui n'est pas seulement kantien. D'une part, et la chose est assez connue et documentée ${ }^{13}$, Barth s'inscrit dans une filiation néokantienne, très exactement marbourgeoise, représentée en philosophie par Hermann Cohen, et en théologie par Wilhelm Herrmann ${ }^{14}$. La pensée des philosophes de Marbourg dans la suite de Cohen peut être caractérisée, en ce qui concerne leur conception de l'expérience, comme une radicalisation de la théorie kantienne. Tout se passe comme si, pour se débarrasser de l'expérience comme promesse et comme slogan, Cohen avait cherché à en extirper toute prétention à donner quoi que ce soit, jusqu'à la réduire entièrement (du moins dans les dernières éditions de sa Kants Theorie der Erfahrung) à du construit. Alors que pour Kant l'expérience est certes structurée par nos catégories mais n'en est pas moins l'expérience d'un donné, pour Cohen il n'y a plus aucun donné dans l'expérience, ou plutôt le donné lui-même n'est plus qu'un moment dans le processus de construction, c'est encore l'un des concepts que nous utilisons pour organiser l'expérience. Vis-à-vis d'une telle conception de l'expérience, W. Herrmann ne peut qu'être en position difficile: il l'admet pour ce qui concerne les sciences de la nature, voire celles de la culture. En revanche, comment pourrait-il s'en satisfaire en ce qui concerne la théologie? Comment admettre que l'expérience sur laquelle se fonde la théologie, l'expérience religieuse, puisse ne contenir aucun donné et être de part en part construite? Ce serait tout simplement nier la possibilité d'une révélation, ou la comprendre au maximum comme révélation de l'humain à lui-même. D'où l'effort de Herrmann pour définir l'expérience religieuse de manière à sécuriser un espace pour le donné.

Il s'engouffre à cette fin dans une brèche ouverte par la théorie de Cohen elle-même, et très exactement par la manière dont Cohen définit, lui, l'expérience dont il parle, et qui est intégralement construite. Cette expérience (Erfahrung), construite conceptuellement, n'est pas l'expérience en général.

13. Par B. McCormack de nouveau, mais plus directement encore par Friedrich LoHMANN, Karl Barth und der Neukantianismus, Berlin, De Gruyter, 1995.

14. Voir l'étude très claire et pédagogique de Simon FiSHER, Revelatory Positivism? Barth's Earliest Theology and the Marburg School, Oxford, Oxford University Press, 1988, en particulier le chapitre 3. On se reportera aussi avec intérêt au travail de Christophe Chalamet, Théologies dialectiques: aux origines d'une révolution intellectuelle, Genève, Labor et Fides, 2015. 
Cohen la distingue très fréquemment de l'expérience de la conscience psychologique individuelle, à laquelle il ne s'intéresse pas, notamment, ditil, parce qu'elle appartient à tout le monde, alors qu' « en ce qui concerne la manière dont l'esprit procède par rapport aux choses », ce ne sont pas « les enfants et les sauvages [sic] qui constituent les références véritables, mais ceux qui produisent la science $»^{15}$. L'expérience dont la tâche du philosophe est de déterminer les modes de constitution, cette expérience de laquelle tout donné est expulsé, n'est pas l'Erlebnis, l'expérience vécue de l'individu. C'est l'objet de la science.

À l'inverse, c'est ce vécu individuel que Wilhelm Herrmann va utiliser comme fondement de la connaissance théologique. En sorte que, dans le cadre néokantien accepté par Herrmann, où toute l'expérience scientifique est construite, il puisse subsister une expérience religieuse susceptible d'être porteuse d'une révélation donnée, dans laquelle l'esprit soit effectivement mis en rapport avec une extériorité. Mais cette expérience n'est expérience d'un donné que dans la mesure où elle est irréductible à l'expérience scientifique, c'est-à-dire dans la mesure où elle est une expérience absolument singulière, individuelle, privée et au bout du compte, inexprimable. De là ce que l'on appelle le dualisme de W. Herrmann: d'un côté, des sciences de la nature qui s'occupent d'une expérience universelle, construite à partir des lois générales de la pensée humaine; de l'autre, une théologie fondée sur l'expérience d'un donné, mais qui n'est jamais donné qu'à telle ou tel, sous une forme absolument singulière et par conséquent inaccessible à toute saisie scientifique.

Sur ce fond, Barth se livre à une opération conceptuelle assez étrange. En un certain sens, il revient à $H$. Cohen contre W. Herrmann, par une critique extrêmement sévère de l'idée de donné pour penser la révélation, dont il écrit qu'elle « ne constitue pas une donnée psychologique, historique, cosmique, naturelle, ni non plus une donnée d'un rang suprême $\rangle^{16}$. La révélation n'est pas une donnée, en tout cas pas au sens courant, pas au sens de ce que l'on appelle habituellement une donnée ${ }^{17}$. Et pourquoi? Parce que

15. Hermann CoHEN, op. cit., p. 62/92.

16. Karl BARTH, Der Rö̈nerbrief $\left(1922^{2}\right.$ ), Zollikon - Zürich, TVZ, 1940, p. 96; L'Épître aux Romains, trad. Pierre Jundt, Genève, Labor et Fides, 1972, p. 72.

17. On voit ici la difficulté de la position tenue par Bruce McCormack, lorsqu'elle est entendue comme une solution au problème de l'expérience, et non son énoncé. En renvoyant, sans plus de précision, la possibilité d'un donné au « miracle » de l'action de Dieu dans sa 
toutes les choses que nous appelons habituellement des « données », en fait, n'en sont pas. Ce sont des constructions. Barth retrouve et radicalise le principe néokantien de l'unité de l'expérience reposant sur l'unité de notre pensée, et il en resserre encore les mailles puisqu'il lui donne une emprise non seulement sur l'expérience entendue comme objet de la science, mais également sur l'expérience intime et privée, celle que Herrmann réservait à la théologie et qui permettait d'en faire une connaissance distincte des sciences de la nature. Il ne saurait donc exister chez Barth de régime d'exception pour l'expérience religieuse - entendue ici au sens de l'expérience religieuse véritable, l'expérience de la révélation.

Qu'est-ce qui autorise Barth à faire cette radicalisation et à étendre le constructivisme cohénien jusqu'aux expériences individuelles? J'ai suggéré l'hypothèse ${ }^{18}$ que s'il le peut, c'est au prix d'une hybridation conceptuelle par laquelle il adjoint au constructivisme de Cohen l'épistémologie nietzschéenne du fait comme interprétation. Barth n'a pas connu le fragment de Nietzsche: «Contre le positivisme, qui en reste au phénomène, "il n’y a que des faits", je voudrais dire: non, il n'y a précisément pas de faits, seulement des interprétations $»^{19}$. Cependant, il a lu Par-delà bien et mal, où Nietzsche déploie cette même épistémologie. Barth la noue au constructivisme de Cohen, pour substituer aux lois universelles (et essentiellement mathématiques) de l'esprit humain des récits interprétatifs qui deviennent les matrices de la construction de l'expérience. Or ces récits, à la différence des lois mathématiques de la pensée, peuvent être des récits individuels ou qui prétendent avoir prise sur les expériences individuelles, des narrations singulières: si bien que ce n'est pas seulement l'expérience de la science, mais toute expérience, y compris la plus singulière, qui peut toujours être

révélation, il reste envisageable de comprendre l'expérience barthienne de la foi sur un modèle herrmannien: comme un sentiment positif bien qu'ineffable, une expérience de plus (mais inanticipable et dont les conditions de possibilités sont inexprimables). Pour aller plus loin et distinguer plus nettement Barth de Herrmann, il faudrait mieux spécifier l'expérience de la foi par rapport à l'expérience en général. Ce n'est pas nier son caractère miraculeux, mais définir plus précisément en quoi consiste le miracle, qui n'est pas simplement une expérience de plus chez Barth mais, on le verra, plus qu'une expérience.

18. Dans mon article «A Philosophical Audacity: Barth's Notion of Experience Between Neo-Kantianism and Nietzsche », International Journal of Systematic Theology, vol. 17, $\mathrm{n}^{\circ}$ 1, 2015, p. 68-88, DOI: 10.1111/ijst.12077.

19. Friedrich NIETZSCHE, Nachgelassene Fragmente Ende 1886 - Frühjahr 1887, Digital critical edition of the complete works and letters, based on the critical text by Giorgio Colli and Mazzino Montinari, Berlin - New York, De Gruyter, 1967, éd. par Paolo D’Iorio, NF$1886,7[60]$. 
soumise à interprétation. Et au bout du compte, l'unité de l'expérience tient dans son universelle ambiguïté, dans son vieldeutiger Charakter, soit la possibilité, en droit, de toujours opposer une interprétation à une autre:

Qui nous délivrera de l'impression irrésistible que toutes ces choses sont intimement enchevêtrées? Qui nous délivrera du soupçon, frisant la certitude, que mon histoire et que l'histoire du genre humain pourraient bien, en définitive, être écrites avec plus de sincérité et de fidélité du point de vue de l'estomac, etc., que du point de vue de la tête et des têtes? Qu'est-ce que l'histoire du monde, si le christianisme primitif, les croisades et la Réforme peuvent être expliqués, du point de vue du matérialisme historique, mieux ou en tout cas d'une manière plus plausible, que de toute autre manière ${ }^{20}$ ?

La Dogmatique généralise encore davantage:

Y a-t-il aussi un seul phénomène dit religieux qui ne se laisse interpréter plus ou moins heureusement et complètement comme prototype ou résidu d'une tentative tout à fait terrestre de donner sens à la vie, comme un phénomène de travail au sens le plus large du terme? [...] Où sont les limites de la possibilité de tout interpréter en termes de religion en général, de culture, d'humanité ordinaire et finalement aussi de biologie ${ }^{21}$ ?

Voilà pourquoi au bout du compte l'expérience ne peut tenir sa promesse. Elle est entièrement plane, sans relief ou sans profondeur permettant d'établir le contact avec l'extérieur. Et le plan est celui de notre pensée, de la multiplicité de nos récits interprétatifs, sans point d'échappée.

\section{Plus qu'une expérience}

J'ai pourtant annoncé que Barth, contrairement à Cohen qui ne conserve la notion d'expérience qu'en la démonétisant entièrement, ne renonce pas, lui, à la promesse de l'expérience. Au contraire, il en fait la clef de voûte de la connaissance théologique. Comment est-ce possible? Sa réponse tient en une formule: l'expérience religieuse véritable, soit la foi, c'est-à-dire la relation subjective à l'acte de révélation de Dieu comme effectivement donné, " n'est pas une expérience, elle est plus qu'une expérience [nicht Erfahrung, mehr als Erfahrung] $\gg^{22}$.

20. Karl BARTH, L'Épittre aux Romains, op. cit., p. 212/299.

21. KD I/1*, § 6, p. 228/210-211.

22. KD I/1*, $§ 6$, p. $218 / 202$. 
Que la foi ne soit pas une expérience, rien de plus facile à entendre, compte tenu de tout ce qui précède. La foi ne peut pas être une expérience puisque l'expérience n'offre jamais aucun donné extérieur à nos constructions interprétatives. Certes, mais alors aucune connaissance de la révélation n'est possible, puisqu'une telle connaissance, c'est aussi ce que dit Barth, est impossible sans expérience: il faut bien que la révélation nous soit donnée pour qu'elle nous soit connue. Dire que la foi n'est pas une expérience ne suffit donc pas, sauf à renoncer à la théologie. La foi n'est donc pas simplement une non-expérience, mais plus qu'une expérience. Qu'estce que cela signifie?

À ce point, seules des hypothèses sont possibles. Avant d'en proposer une, il vaut la peine de souligner la grande singularité du passage en question dans l'œuvre de Barth. Le théologien est d'ailleurs conscient de cette singularité, qui vient notamment du fait que son identification de l'expérience religieuse véritable à la foi, et donc de la foi à une expérience, va contre l'interprétation la plus habituelle de sa pensée ${ }^{23}$. D'autant que pour caractériser cette identification, Barth fait appel, à ma connaissance pour la seule fois de manière aussi claire dans son œuvre, à la « conceptualité mystique »-s'autorisant toutefois pour cela de Luther et Calvin ${ }^{24}$. Et c'est là, à mon avis, une indication décisive: c'est en gardant à l'esprit cette référence à la conceptualité mystique qu'on peut entendre au mieux ce que signifie le «plus » du " plus qu'une expérience ». Car on peut alors le rapprocher d'un autre " plus », fréquemment de mise dans cette conceptualité mystique et d'abord chez Denys l'Aréopagite, qui parle notamment de Dieu comme de ce qui est au-delà, au-delà de l'essence par exemple.

23. Voir ibid., p. 219/203: « Je suis forcé d'insister là-dessus, souffrant d'entendre répéter sans cesse que je cherche à enseigner une fides quae creditur "sans me préoccuper de la fides qua creditur, de la conviction de foi personnelle et de l'expérience de la foi" ». Barth mentionne ici Wobbermin, mais c'est une idée encore largement répandue autour de sa pensée.

24. KD I/1*, §6, p. 232/215. Comme je l'ai remarqué ailleurs, c'est aussi la seule référence à la mystique oubliée par Jean-Louis Leuba dans son étude pourtant très complète « Mystique et barthisme » (dans Jean-Louis LeubA, Études barthiennes, Genève, Labor et Fides, 1987, p. 97-138), qui par conséquent n'envisage pas d'autre rapport de Barth à la mystique que critique. Les traducteurs de la Dogmatique, quant à eux, ont traduit «mystische Begrifflichkeit » par «abstraction mystique », ce qui semble injustifiable sinon par une impossibilité, là aussi, d'envisager une valorisation positive du terme par Barth. Barth est en effet, et encore aujourd'hui, considéré le plus souvent comme un théologien anti-mystique (voir par exemple Frédéric Nef, La connaissance mystique, Paris, Cerf, 2018, p. 24: « la théologie dialectique de Karl Barth refuse la mystique »). 
Or cet au-delà dionysien, le suressentiel ${ }^{25}$, plus qu'essence, signifie quelque chose comme une positivité de la négation en elle-même: l'essence est niée en tant qu'essence, mais cette négation n'est pas purement négative, elle ne renvoie pas Dieu à de l'inessentiel ou du non-être. La négation ellemême est une indication positive quoique jamais formulable de ce qu'il est, et c'est ce que signifie le « suressentiel » : non pas autre chose que l'essence, qui serait positivement énonçable d'une autre manière et tel qu'on pourrait abandonner le terme d'essence, comme si celui-ci n'avait rien à nous dire de Dieu, mais une certaine forme de négation de l'essence elle-même, qui est en elle-même l'indication de la réalité de Dieu parce qu'elle indique une certaine manière pour lui de n'être pas essence tout en étant quelque chose de l'essence, et même l'essentiel de l'essence, qui ne peut plus se dire en termes d'essence mais pas non plus autrement.

Qu'en est-il, si l'on transpose ce raisonnement à la phrase de Barth: « non pas une expérience, mais plus qu'une expérience »? Il faut alors non pas séparer les deux membres de la phrase: d'abord une négation (nicht Erfahrung), puis une affirmation (mehr als Erfahrung), comme si Barth écartait d'abord l'expérience, pour introduire ensuite autre chose. La formule doit se comprendre d'un bloc, le plus n'étant qu'une manière de comprendre la négation de l'expérience elle-même, et comme une manière non pas de s'éloigner de l'expérience mais comme la seule manière possible d'indiquer ce qu'il en est de l'expérience véritable. De même qu'on ne comprend l'essence de Dieu qu'en niant qu'il soit une essence - il est plus qu'essence, suressence -, de même on ne comprend ce qu'est l'expérience qu'en la niant, et cette négation est la foi. Autrement dit, cela signifie que la foi est plus qu'expérience précisément en tant qu'elle n'est pas expérience. Plus concrètement encore, cela veut dire que ce que donne l'expérience religieuse véritable, la foi, ne peut être autre que la conscience de l'impossibilité de toute expérience religieuse véritable: sa propre négation ou son propre échec.

C'est déjà ce qu'affirmait Barth en 1922 dans le chapitre de L'Épître aux Romains consacré à l'expérience religieuse. Elle y est décrite non pas comme un point d'ouverture dans l'expérience humaine générale, par lequel

25. Le concept est aussi fréquent dans la mystique rhénane, ce qui nous rapproche déjà de Luther et donc de Barth. Voir à ce sujet Mariel MAzzocCO, "Suressentiel". Aux sources d'un langage mystique », Revue de l'histoire des religions, 4 | 2013, p. 609-627, DOI: $\underline{10.7202 / 1039223 \mathrm{ar}}$. 
la révélation de Dieu se donnerait comme quelque chose de radicalement différent, mais tout au contraire comme « le point de fermeture et, pour cette raison même, secrètement ouvert dans le cercle de l'humanité ». Si ouverture il y a, c'est dans et par la clôture du cercle, tout comme s'il y a plus qu'expérience, c'est dans et par l'absence d'expérience. La religion révèle la clôture de l'expérience humaine sur elle-même :

Qu'en est-il du reste de ses possibilités, si cela [la religion] constitue leur pointe, leur pointe ultime, leur pointe la plus profonde? $\mathrm{Si}$, spécialement la justice humaine la plus haute est... sacrilège? [...] Avec le maillon terminal, la chaîne entière se révèle comme une série d'impossibilités. [...] La maladie qui conduit à la mort éclate ${ }^{26}$.

Toutefois si dans l'expérience religieuse ne s'opère aucune sortie du domaine habituel de l'expérience, d'où vient le plus? D'où vient qu'elle donne malgré tout quelque chose et réalise ainsi la promesse de l'expérience? Tout simplement du fait que sans elle, sans l'expérience religieuse véritable, c'est-à-dire sans la foi, on ne pourrait même pas savoir que l'expérience est absolument plane, que toutes nos expériences se tiennent dans un cercle fermé: " le lieu, d'ou il faut voir, comme tel, le cercle tout entier, ce cercle fermé, ne peut se situer, lui-même, au-dedans de ce cercle.». C'est pourquoi je disais que la formule de Barth devait s'entendre d'un seul tenant: « non pas expérience, plus qu'expérience » ne veut pas dire: "pas expérience mais plus et donc autre chose », mais " pas expérience c'est-àdire plus qu'expérience »- ou sur-expérience. Ou encore: l'expérience religieuse, la foi, accomplit la promesse de l'expérience en tant qu'elle la manifeste justement comme détresse. Ce que Barth exprimera de nouveau en 1939 à l'aide d'un vocabulaire plus nettement théologique encore: « c'est si entièrement par la seule grâce de Dieu que la vraie religion est la vraie, qu'elle doit se laisser démasquer et condamner par la grâce comme fausse religion $»^{27}$.

Car le fait que toute notre expérience soit manifestée comme inéluctablement fermée sur elle-même, bien que cela soit strictement négatif, n'est pas rien (non pas seulement pas mais plus). C'est même beaucoup, puisque justement, la plupart du temps, nous avons l'impression de faire des expériences positives de révélations. Nous avons l'impression de ne pas être

26. Karl BARTH, L’Épître aux romains, op. cit., p. 254/261 (trad. modifiée).

27. KD I/2**, § 17, p. 361/119. 
enfermés dans le cercle de nos expériences humaines. Autrement dit, nous croyons que nos expériences sont effectivement des lieux où le réel se donne, où Dieu lui-même peut parler. Et la foi consiste d'abord, pour Barth, à ne plus y croire: la révélation est d'abord la révélation du fait que nous ne voyons rien. Elle est l'expérience de l'impossibilité pour l'expérience de rien donner.

Qu'est-ce à dire, sinon que la foi tient dans l'ambivalence de l'expérience? Non pas, il faut y faire attention, dans l'expérience de l'ambivalence (car cette expérience, justement, ne serait pas elle-même ambivalente) mais dans l'ambivalence de l'expérience. Non pas dans une contre-expérience au sens d'une expérience spécifique et singulière, différente de toute autre expérience, mais dans le fait que l'expérience apparaisse comme elle est, construite par nos pensées, via nos multiples interprétations en conflit.

Si la foi tient dans l'échec de nos expériences, aucune expérience ne peut jamais attester de la foi. Cela signifie que le caractère religieux d'une expérience, le fait qu'un état mental vécu puisse être caractérisé comme foi, n'appartient à aucun vécu en tant que tel. Mais cela signifie en même temps, et pour la même raison, que tout mental vécu peut être caractérisé comme foi, sans qu'il cesse par là même d'être ambigu, justement parce que c'est cette ambiguïté qui en fait une expérience de foi.

Voilà donc ce que fait Barth de la tension dont je parlais au début et qui travaille la notion d'expérience: sa détresse et sa promesse. Il en fait le cœur de l'expérience religieuse, qui devient l'expérience par excellence, mais justement parce qu'elle ne triomphe pas de l'ambiguité de l'expérience, de sa détresse, mais la magnifie au contraire. Étant l'échec de l'expérience, elle est par là-même sa véritable réussite, le seul vrai donné, tout ce qu'elle peut donner, à savoir la manifestation de son incapacité à donner.

\section{Et après? Philosophie et théologie catholique barthiennes}

La question, toujours la même lorsqu'il s'agit de Barth, est évidemment de savoir: que faire de tout cela? À quoi nous mène la conception barthienne de l'expérience religieuse, que peut-on construire ou reconstruire sur une telle base, comment peut-on continuer à être barthien, avec un tel point de départ? Et il s'agit évidemment d'une mauvaise question. En effet, si les constructions théologiques ne sont des connaissances qu'en tant qu'elles sont aussi des expériences, c'est-à-dire dans la foi, mais si ces expériences ne 
peuvent elles-mêmes être déterminées a priori, circonscrites à certains phénomènes, mais tiennent au bout du compte dans un (in)certain tremblement de nos expériences, alors il serait illusoire de vouloir que la théologie progresse à la manière d'une science positive, fondée sur certains principes ou certaines données stables, indépendantes de la situation dans laquelle chaque théologienne ou théologien est engagé. La pensée de Barth ne saurait être un manuel de théologie pour tous les temps, et même pas pour le nôtre: " "Merci de cette promesse dont je ne peux faire l'expérience que comme d'une détresse!" " , cite Barth malicieusement dans la transcription écrite de l'une de ses conférences - serait-ce qu'on lui avait déjà posé la question directement? « - et je n'aurai rien à rétorquer $»^{28},(\mathrm{se})$ répond-il.

On pourrait tout de même souligner une conséquence possible de sa manière de penser l'expérience religieuse, que je formulerai d'abord en utilisant un langage davantage épistémologique, puis plus sensiblement théologique.

En ce qui concerne la théorie de l'expérience, Barth invite à penser que l'intensité d'une expérience, ou plus exactement sa profondeur, sa signifiance, c'est-à-dire sa valeur de promesse, n'est non seulement pas incompatible avec, mais intrinsèquement liée à son ambiguïté. Une expérience n'est pas moins éprouvée, mais plus, parce qu'elle est ambiguë. La question serait de savoir quelles catégories devraient être utilisées pour saisir ce paradoxe apparent, c'est-à-dire pour découpler la certitude de, par exemple et pour emprunter une distinction à Étienne Gilson, l'évidence ${ }^{29}$. Car la foi chez Barth est une évidence non certaine, et dont l'évidence n'est pas en raison de sa certitude. On peut tout à fait voir sa vie bouleversée, convertie, par une expérience dont on peut en même temps continuer de douter. Et la transformation de la vie elle-même peut continuer d'être ambiguë. Mais justement la saisie de cette ambiguité pourrait être le mode même sous lequel se donne l'évidence de la foi. L'erreur philosophique initiale des partisans du slogan moqué par $\mathrm{H}$. Cohen fut peut-être de croire que l'expérience allait pourvoir la métaphysique de la certitude qui lui manquait. C'était peut-être rester encore trop cartésien, et ne pas aller au bout du mouvement que le recours à l'expérience permettait de commencer en abandonnant la perspective de fondations purement rationnelles de la

28. Karl BARTH, Parole de Dieu et parole humaine, op. cit., p. 220.

29. Voir Étienne GILSON, Réalisme thomiste et critique de la connaissance, Paris, Vrin, 2012, p. 47-48. 
connaissance. En réalité peut-être l'expérience ne peut-elle donner ce qu'elle a qu'en nous faisant abandonner la certitude elle-même - pour l'évidence. Un tel déplacement ne serait pas sans importance pour essayer d'avancer dans le dépassement de ce qui peut aujourd'hui apparaître comme l'aporie cartésienne et même phénoménologique: la recherche de la première certitude. L'expérience peut être fondatrice sans être certaine, parce que l'ambiguïté, le doute, pourrait être le mode de donation de la plus grande certitude. Voilà ce que suggère Barth, parce que ce que manifeste l'évidence, c'est précisément notre finitude et par là notre incapacité à la certitude: il y aurait une contradiction à connaître de manière certaine notre finitude.

Derrière ces questions épistémologiques et phénoménologiques, que je viens de décrire sommairement et pour seulement lancer quelques pistes de recherche, puisque c'est le rôle du séminaire dont ce travail est issu, se profile peut-être une question plus explicitement théologique, qui pourrait faire penser qu'il reste malgré tout quelques ressources dans la pensée de Barth, non seulement pour la philosophie mais pour la théologie ${ }^{30}$, y compris catholique. Car il y a en français un deuxième sens de l'expérience, lié au premier mais qu'une concentration unilatérale sur le sens kantien du mot peut faire oublier. L'expérience n'est pas seulement l'ensemble de mes expériences possibles, mais aussi l'accumulation de mes expériences effectives, la sédimentation de mes expériences vécues, comme lorsque l'on dit d'une personne qu'elle a de l'expérience. Et la spécificité de l'expérience de foi telle qu'en parle Barth obligerait presque à poser une autre question, relativement à ce deuxième sens, celle de savoir si l'on peut être expérimenté dans la foi. Or à cette question, d'après tout ce que l'on vient de voir, il faudrait répondre: oui, on le peut, on peut accumuler, individuellement et collectivement, les expériences de foi, et cette accumulation est effective. Mais il ne peut s'agir d'expériences de foi que dans la mesure où elles sont ambiguës, si bien que leur accumulation implique une augmentation tout à fait corrélative de cette ambiguïté. Ou en d'autres termes, on peut acquérir de l'expérience dans la foi mais cette acquisition n'augmente pas la certitude qui y est attachée. Peut-être, en revanche, gagne-t-elle en évidence, mais d'une évidence incertaine, on l'a dit, quoique pas moins évidente d'être

30. Sur la reprise possible de Barth en philosophie et en théologie, ses modalités et le rapport entre les deux gestes, je me permets de renvoyer à ma thèse publiée sous le titre $L e$ serpent d'Aaron. Sur l'expérience religieuse chez K. Barth et H. Bergson, Lausanne, L'Âge d'Homme, 2015. 
incertaine. On ne peut donc pas éviter de se référer à ces expériences accumulées, mais on ne peut pas les considérer comme les étapes d'un chemin linéaire ou les marches d'un escalier vers l'accomplissement d'une foi de plus en plus certaine (et de moins en moins croyante?). Elles ne gagnent leur évidence qu'à être réinterrogées, une à une et en totalité. Des expériences décisives mais incertaines et par conséquent à interroger, mais qui ne sont pas moins décisives parce qu'elles sont incertaines, ne serait-ce pas ce que l'on appelle une tradition?

Anthony FENEUIL 